\title{
Infection Control and Hospital Epidemiology: Transitioning Through Adolescence!
}

\author{
William R. Jarvis, MD
}

As we begin 2006, it is a fitting and appropriate time to provide an overview of the current status of the journal of the Society for Healthcare of America (SHEA), Infection Control and Hospital Epidemiology (ICHE). This is an exciting time. With this issue, a major transition in the growth of our journal is taking place: the University of Chicago Press (UCP) has become our publisher (welcome Gordon Rudy, Everett Conner, and staff). With this transition in publisher, it is a good time to review where we have been and, most importantly, to discuss how we have changed and the exciting and bright future that we have.

First, I would like to review selected aspects of our past, which illustrate why this publisher transition is such a sentinel event for the journal. Since the initiation of ICHE through the vision of Dr. Richard Wenzel, Slack Incorporated has been our publisher. Slack has supported us during good times and bad over these decades. They have helped us through 3 editor transitions: from Dr. Wenzel to Dr. Michael Decker to Dr. Barry Farr to me. In addition, Slack has provided strong support for the journal during times of difficulty; SHEA and our journal, like all journals, have had many growing pains. I would like to thank John Carter and Richard Roash for their constant and unending support over these years. As or even more importantly, I would like to thank Shirley Strunk, Sheryl Croce, and Jaime Underwood at Slack. Those of you who have submitted or reviewed an article for the journal know that these 3 people have been critical to the success of our journal. They have been the interface between the author, editor, reviewer, publisher and SHEA-literally, the glue that holds ICHE together. I would like to thank them for their unending dedication, hard work, enthusiasm, and support. Their excitement about our journal has been contagious and has led to significant improvements in the quality of $I C H E$ over the past decades.

Second, I would like to review the latest editor transition. In June 2004, I joined Dr. Farr as Deputy Editor of the journal, at a time when Dr. Farr was retiring from the University of Virginia and deciding whether he would be able to continue as the Editor of ICHE. Over the next 6 months, I had the pleasure of working closely with Barry on editing the journal.
This was an invaluable lesson for me. It is hard to describe the level of Barry's dedication to ICHE. During his tenure as Editor, Barry made enormous strides in advancing our journal. The number of articles submitted increased, article processing times dramatically decreased, the journal impact factor increased, and the quality of the articles published and the format of the journal (ie, the introduction of theme issues) dramatically improved. In addition, Barry was a fantastic editor who for years spent virtually all of his "free" weekend (and other) time editing the journal. Barry loved ICHE, and it showed. It was with deep regret that he decided he could no longer continue as the Editor. In January 2005, the SHEA Board named me Editor. I thank the SHEA Board for their vote of confidence in me. I have enormous shoes to fill and just hope I can approach Barry's achievements. I thank all the previous editors for their dedication and sacrifices for $I C H E$, and I look forward to building upon their legacy.

Third, let's take a look at the evolution of the journal over the past several years. Having transitioned through infancy and childhood, ICHE clearly is in its adolescence. We are witnessing an enormous growth spurt in the number of submissions. In 2002 and 2003, the same number of articles was submitted. In contrast, in 2004, the number of manuscripts submitted increased by $27 \%$ (compared with 2002 or 2003 ). In 2005 (through November), the number of submissions has increased over 2004 by an additional 19\%. Thus, in the past 2 years, the number of submissions has increased by almost $50 \%$ ! Needless to say, this has stretched our capacity, as it would for any journal. All journals struggle to obtain timely reviews of submitted articles. We try to insure that we identify reviewers who are knowledgeable about the specific subject of each manuscript, that they are thorough and fair, and that they review the submissions as promptly as possible. To all of our dedicated reviewers, I would like to thank you for your continued support of ICHE. Given the large increase in the number of submissions, additional qualified reviewers are needed so that we do not increase the burden of reviewing on our existing reviewer pool. The longest delay in processing submitted articles is incurred while we identify qualified reviewers with the time to review the paper. Often, we com- 
municate with 5-10 potential reviewers before identifying 2 reviewers who have the time and interest to review the manuscript. If you would like to review manuscripts for the journal, please contact either me or our UCP personnel.

The large increase in submissions also has led to a second problem: a large backlog of accepted but unpublished articles. To those authors caught in this situation, I apologize. We are doing all we can (given the terms of the Slack and now UCP contracts) to quickly reduce this backlog. During the recent 2-year period of increasing submissions, the rejection rate remained relatively stable. During part of 2005, Slack generously published additional pages in each issue; again we thank them. Now, our contract with UCP includes additional pages for each issue and we are investigating additional options that will allow us to publish these accepted papers even more rapidly. Unfortunately, another necessity given our situation is that the rejection rate for our journal will have to increase. It has increased in 2005 and will have to increase even further in 2006. Our reviewers will have to be even more critical and recommend acceptance of an even smaller number of submitted papers. Since a large increase in submissions has been from our international authors, we are trying very hard to insure that this increased rejection rate does not disproportionately impact those in the field of healthcare epidemiology in countries with limited support or resources. We are an international society and encourage submission of papers from our colleagues throughout the world (additional reviewers from outside the United States also are needed).

Last, let me look to the future. This issue, with its somewhat new Editor, new publisher, new format, and increased number of articles, begins a new era in the growth cycle of $I C H E$. We hope you like the changes. More changes are to come. I welcome UCP as our new publisher. We have a very strong, dedicated, and experienced UCP staff with new and inno- vative ideas for improving our journal. Over the next year, there will be many changes, some visible and some not so visible- - but all done to enhance the quality and function of the journal. For example: we will add many more qualified reviewers to our already large reviewer pool, send papers for review within 1-3 days of receipt, strive to obtain reviews as quickly as possible, decide on the status of papers within 13 days of receipt of the review (so that the majority of the time from submission to acceptance is accounted for by peer review and the authors' revision of the manuscript), solicit meta-analyses and review articles, expeditiously publish the most important papers that enhance the science of healthcare epidemiology, rapidly reduce our backlog of unpublished manuscripts, develop editorial board guidelines and revise its membership, and reexamine all aspects of our journal format (eg, the use of sections) and processes to insure that we maintain the legacy developed by previous editors of ICHE-the preeminent source for the best science on healthcare epidemiology and infection prevention and control.

I and UCP are interested in hearing from you about how we can further enhance the journal. I urge all SHEA members (and others) to let us hear from you about what journal enhancements you would like. I thank all authors who have shown confidence in us by submitting manuscripts to us. I urge all authors to submit their best science to ICHE. We will do everything that we can to process your submission as promptly as possible and to insure prompt publication of all articles. In addition, we have developed a special "fast track" for those exceptional manuscripts that represent the very best science in the field, so that we can publish these articles more rapidly than competing journals can. I and UCP will do everything that we can to insure that we guide our rapidly growing adolescent $I C H E$ into a strong adult. 